\title{
Teaching Fundamental Aspects of Natural and Artificial Photosynthesis in Higher Education
}

\author{
Urs Leisinger ${ }^{\mathrm{a}}$, Urs Leutenegger ${ }^{\mathrm{a}}$, and Roger Alberto ${ }^{\mathrm{b} *}$
}

\begin{abstract}
Photosynthesis or the storage of solar energy in chemical bonds is a process which, in its essence, goes far beyond a conversion of $\mathrm{CO}_{2}$ and water into dioxygen and glucose or other organic products. Photosynthesis is a process which comprises elementary features of most of the chemical reactions and some physical processes we are looking at in higher education; light absorption, proton transfer, redox reactions and making and breaking of bonds amongst others. Metals and main group elements are involved and the entire process is embedded in a biological environment that involves proteins and membranes. In this article, we will focus on two key aspects of natural photosynthesis, namely the absorption of light (photons) and the separation of the excitons into electrons and holes based on P680 along with the electrochemical energetics. Further, we will present an artificial light driven catalytic process which mimics PSI for the reduction of water to $\mathrm{H}_{2}$, the inorganic congener of nicotinamide adenine dinucleotide phosphate (NADPH). This light-driven reductive process shall show the mechanistic complexity of the 'easy' reaction $2 \mathrm{H}^{+}+2 \mathrm{e}^{-} \rightarrow \mathrm{H}_{2}$.
\end{abstract}

Keywords: Biomimetic · Electron transfer reactions · Excited states · Photosystems I and II · Reaction mechanisms $\cdot$ Water oxidation and reduction
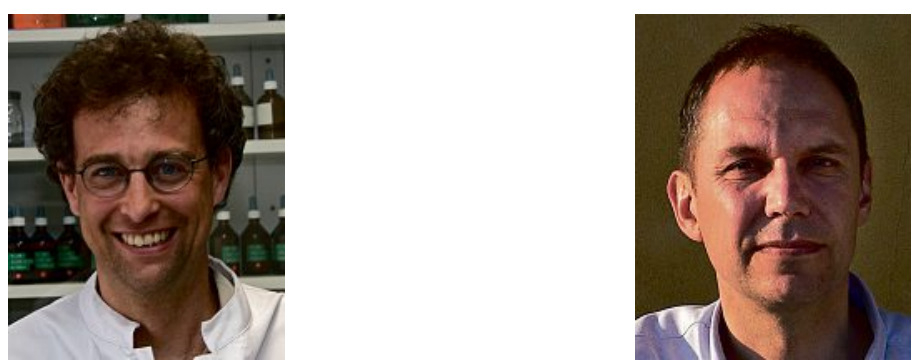

Urs Leisinger studied environmental sciences at the Swiss Federal Institute of Technology in Zurich (ETH), including a practical semester at the Plant and Pollution Research Laboratory in Tallinn, Estonia. He received his $\mathrm{PhD}$ for work on the response to oxidative stress in green algae under the supervision of Rik Eggen, Eawag Dübendorf, in 2001. In 2004, he obtained his Certificate in Education at the ETH and since 2001, he works as a chemistry teacher at the Kantonsschule Zug.
Urs Leutenegger obtained his Diploma in Natural Sciences at the ETH Zürich in 1985. He received his $\mathrm{PhD}$ in Organic Chemistry under the supervision of Prof. A. Pfaltz at the ETH Zürich in 1990. His research work focused on enantioselective catalysis with semicorrin-transition metal complexes. He joined the group of Prof. W. C. Still at Columbia till summer 1990 before working for Basler and Hoffmann, an engineering company in Zürich. In 1993 he obtained his Certificate in Education from the ETH. After teaching chemistry at the Kantonsschule Rychenberg in Winterthur, he moved to the Kantonsschule Zug, where he has been teaching chemistry since 2000 .

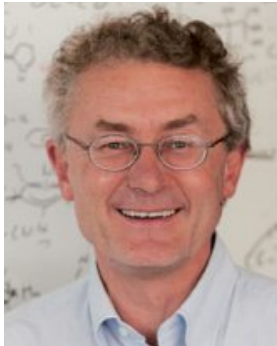

Roger Alberto studied chemistry at the ETH Zürich culminating in a $\mathrm{PhD}$ under the supervision of Prof. G. Anderegg on technetium fluorides in 1988. After postdoctoral stays as an Alexander von Humboldt fellow with Prof. W. A. Herrmann at the TU Munich and Prof. A. Sattelberger at Los Alamos National Laboratory (LANL) 1989-91, he became a group leader at Paul Scherrer Institute (PSI) from 1992-1999, associate Professor at the University of Zürich in 1999 and finally full professor in 2005. He has undertaken guest professorships in Sendai Japan, Paris and Singapore. From 2012-2016 he was head of the Department of Chemistry. His research interests include technetium and rhenium, bioorganometallic chemistry, basic organometallic chemistry in water, field sensor probes for MR imaging. He is the chair of the University Research Priority Program 'Light to Chemical Energy Conversion' (LightChEC) and was awarded the Alexander von Humboldt research award in 2017. 


\section{Introduction}

Natural photosynthesis in plants is a process in which diluted solar light energy is converted into concentrated chemical energy. Ultimately, photocatalysis provides all the energetic compounds that are needed to drive our society. It is a highly complex and multi-step biochemical process which can be generally described as the absorption of photons and the storage of their energy in chemical form. In the best-known oxygenic photosynthesis of cyanobacteria, algae and higher plants, the final products are essentially dioxygen $\mathrm{O}_{2}$, NADPH and ATP as intermediates for the glucose formation and glucose $\mathrm{C}_{6} \mathrm{H}_{12} \mathrm{O}_{6}$ itself. ${ }^{[1,2]}$ The initial step of photosynthesis in the so-called reaction centres is photoninduced charge separation, generating reducing equivalents in the form of electrons and simultaneously oxidizing equivalents in the form of holes. ${ }^{[3]}$ The reducing equivalents are used to generate organic building blocks for biomolecules from inorganic, stable compounds such as $\mathrm{CO}_{2}$ whereas the latter holes are filled by electrons from water $\mathrm{H}_{2} \mathrm{O}$ oxidation to form the 'waste product' and strong oxidant $\mathrm{O}_{2}$. The overall photosynthesis process can be summarized as:

$$
\begin{aligned}
& 6 \mathrm{CO}_{2}+6 \mathrm{H}_{2} \mathrm{O} \rightarrow \mathrm{C}_{6} \mathrm{H}_{12} \mathrm{O}_{6}+6 \mathrm{O}_{2} \\
& \Delta \mathrm{H}^{\circ}{ }_{\mathrm{R}}=2800 \mathrm{~kJ} \mathrm{~mol}{ }^{-1} \\
& \Delta \mathrm{G}^{\circ}{ }_{\mathrm{R}} \approx 2875 \mathrm{~kJ} / \mathrm{mol}
\end{aligned}
$$

Every hour about $4.2 \cdot 10^{20} \mathrm{~J}$ energy reaches the earth surface, approximately the energy that is turned over by humans in technical processes on our planet in one year $\left(4.1 \cdot 10^{20} \mathrm{~J}\right)$. However, only around $0.1 \%$ of this influx is deposited in form of biomass, approximately equal amounts on land ecosystems and in the oceans, ${ }^{[4]}$ a disputed source of energy for satisfying our energy demand in the future. ${ }^{[5,6]}$ If this amount of solar energy could be converted and stored to a sufficient extent with efficient devices of some kind, energy problems could be solved and the increase of $\mathrm{CO}_{2}$ in the atmosphere be stopped or even reversed. Efficiency is a major issue. It can be high as in wind turbines (Betz law, up to $60 \%$ electric energy with respect to the kinetic energy of the wind) but is mostly in the range of $10-20 \%$ with modern siliconbased photovoltaic cells.

Looking at photosynthesis, we find that the minimal requirements for producing one molecule of glucose are 24 photons at $680 \mathrm{~nm}$ and 24 photons at $700 \mathrm{~nm}$. This light energy adds up to $8600 \mathrm{~kJ} / \mathrm{mol}$ which corresponds to an energetic efficiency of about $30 \%$, as the reaction enthalpy of photosynthesis is $2800 \mathrm{~kJ} / \mathrm{mol}$. $^{[7]}$ If the free enthalpy change $\Delta \mathrm{G}^{\circ}{ }_{\mathrm{R}}$ of the overall photosynthetic reaction is compared to the exergy of the photons, i.e. maximal energy that can be used for chemical work according to the second law of thermodynamics, the recovery is even higher, reaching about $40 \% .[8]$

However, this is theory and for a true efficiency the full incident solar spectrum has to be considered. Light energy losses in the plant start with light scattering in the photosynthetic tissues and elsewhere (approximately 30\%), the radiation that can be absorbed by chromophores is about $53 \%$ (see Fig. 2 below). ${ }^{[7]}$ Moreover, losses of light energy in the photosynthetic antenna due to the funnelling of only about $1.8 \mathrm{eV}$ per photon into photochemical reactions reduce efficiency further by $25 \%$. Including the subsequent chemical fixation with an estimated efficiency of about $30 \%$, accumulated losses total up to an approximate solar energy storage efficiency of $\approx$ $9 \%$. Minimal respiratory losses of $30 \%$ add up, capping the efficiency of biological photosynthesis at a maximum of $6 \%$. This compares to the highest solar energy conversion efficiency reported for crops of about $3.7 \% .[9,10]$ This value includes the energy required to construct and maintain the photosynthetic apparatus, whereas the efficiencies quoted for technical devices normally do not include the energetic maintenance costs. ${ }^{[2]}$

According to these estimations, losses in the photosystems and losses in the subsequent photochemistry reduce the yields each by a factor of approximately 3 . The remaining losses that reduce the yield by a much higher factor, thus, are a consequence of oversaturation of the photosynthetic apparatus with light at radiation intensities above about $10 \%$ of full sunlight, incomplete ground cover and the fact that biological photosynthetic systems are situated in a complex biological context and drive various biological processes. ${ }^{[11]}$ Under non-ideal conditions, photosynthetic efficiency is generally $1 \%$, more realistically about $0.1 \%$ as pointed out by Barber et al. in a very interesting study. ${ }^{[4]}$ It is not an issue of this article to compare natural photosynthetic efficiency with artificial systems, but to learn from it. To improve photosynthetic efficiency was probably not a demand in nature since the available systems are optimized and conditionadapted. ${ }^{[12]}$

Going from natural to artificial photosynthesis, the overall reaction of converting $\mathrm{H}_{2} \mathrm{O}$ and $\mathrm{CO}_{2}$ into $\mathrm{O}_{2}$ and glucose, the concept is reduced to simple water splitting into its elements $\mathrm{H}_{2}$ and $\mathrm{O}_{2}$. Although not immediately obvious, $\mathrm{H}_{2}$ is key for our society since essentially all basic large-scale chemicals such as $\mathrm{NH}_{3}$, methanol $\mathrm{H}_{3} \mathrm{COH}$ and others need $\mathrm{H}_{2}$ as a synthetic component in their industrial preparations. ${ }^{[13]}$ If $\mathrm{H}_{2}$ is available, follow up processes such as Fischer-Tropsch or Sabatier to liquid fuels are well established. Artificial water splitting is a field of intense research and a number of systems making this possible have been published, the artificial leaf being probably the most prominent one.[14] Other assemblies which combine electrolytic water splitting with photovoltaics ${ }^{[15]}$ reach solar to dihydrogen\&dioxygen efficiencies up to $13 \%{ }^{[16]}$ but many of these systems are limited due to photodegradation of their components. There are various possibilities to assemble the components in water splitting, wired and non-wired, homo- or heterogeneous and advantages and disadvantages are discussed with respect to economy, ecology efficiency and other parameters. ${ }^{[17]}$ Research is in most fields still far from providing a long-term stable (years), cheap and efficient water splitting device. Still, from the available systems, we can learn about fundamental chemical and catalytic processes.

In this article, selected basic reaction schemes found in natural photosynthesis mainly in PSII will be discussed on a level useful for higher education in gymnasia. Reduction equivalents, conceptually generated in a water oxidizing system such as PSII, will be applied to artificial, PSI mimicking systems to show how demanding the generation and the understanding of a molecule-based architecture is. It is not the intention of this essay to provide a comprehensive view about the state of research in natural photosynthesis or to summarize the latest achievements in artificial photosynthesis. Rather, fundamental processes shall be discussed as relevant for photochemistry and catalysis.

\section{Aspects of Natural Photosynthesis}

The initial steps of natural photosynthesis essentially rely on two components, photosystem II (PSII) where water is oxidized and photosystem I (PSI) where the electrons from PSII are temporarily stored in form of the reductant NADPH and then used for the reduction of $\mathrm{CO}_{2}$ or as an electron source in various anabolic reactions ('biological $\mathrm{H}_{2}$ '). To get an impression of the complexity of PSII, a sketch is shown in Fig. 1 together with some of its important components.

Among the key steps in PSII and PSI are light absorption and energy transfer. Both protein assemblies comprise a large number of chlorophylls and carotenoids for these two processes (for PSII, see Fig. 1). In a biological context, such light-absorbing dyes are called pigments, irrespective of their solubility, and the light-absorbing structures of the pigments are called chromophores. The pigments of the photosys- 


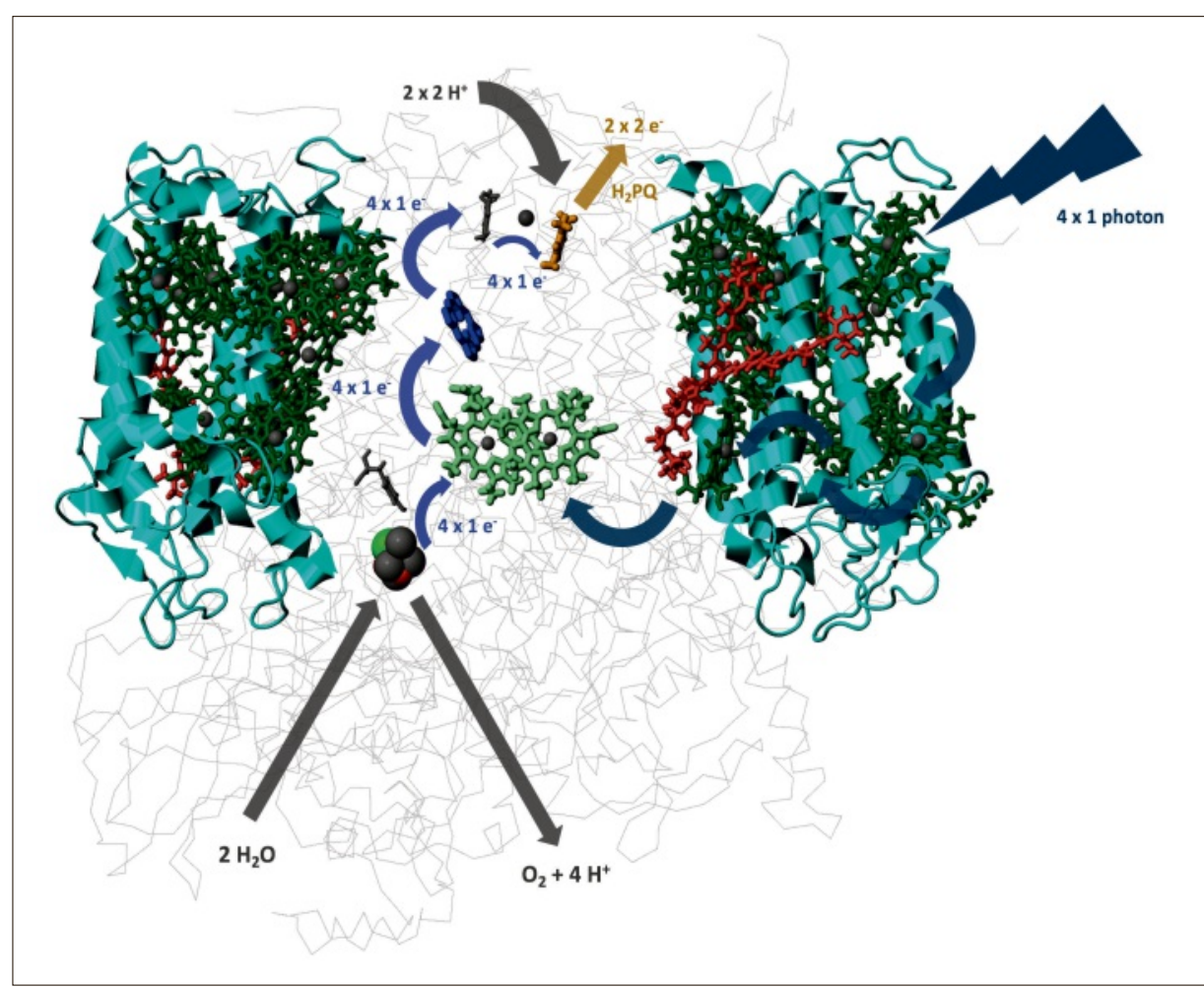

Fig. 1. Core proteins of photosystem II (PSII from Thermosynechococcus elongatus), displayed parallel to the membrane plane with the stroma above. Colour codes: backbone in grey with two associated antenna complexes (CP43 (left) and CP47; ribbon model, cyan). Dark blue arrows display the flow of excitation energy, light blue arrows the transfer of electrons. The shown chromophores are carotenoids (red) chlorophyll (dark green), special chlorophylls of P680 (light green), pheophytin (blue), plastoquinone (orange), water-oxidizing complex WOC (space filling grey, red and green). The positive charge of $\mathrm{P} 60^{+}$resides on the P680-chlorophyll on the left. Chlorophyll and plastoquinone are shown without their isoprenoid side chains. The image was created from crystallographic coordinates found in PDB ID: 1S5L. ${ }^{[18]}$

tems mainly absorb photons from the blue and the red region of the solar spectrum, for which reason vegetation is not a perfect absorber of visible light and leaves are usually green. Other leaf colours account for different absorption wavelengths. Fig. 2 gives a comparison between the solar spectrum and an UV/vis spectrum of pigments found in leaves, e.g. chlorophyll. From this comparison, incompatibility becomes obvious, as green and yellow light is barely absorbed. Chlorophyll molecules show strong absorption bands in two regions of the visible spectrum, the low energy Q bands in the red region and the high energy B bands in the blue region. ${ }^{[19,20]}$ Upon absorption of a 'red photon', chlorophyll gets photoexcited to its lowest excited state with an excitation energy of approximately 1.8 $\mathrm{eV}$. Photoexcitation with 'blue photons', in contrast, yields higher excited states that rapidly relax to the lowest excited state and thereby dissipate the excess excitation energy into heat $(\sim 200 \mathrm{fs}) .{ }^{[20]}$ The lowest excited state lasts for about $4 \mathrm{~ns}$ and thus is sufficiently long-lived to pass on the excitation energy or to reduce electron acceptors and thus qualifies as photosensitizer. ${ }^{[21]}$ This 'downgrading' from higher to lower excited states leads to heat dissipation of around $25 \%$ from the incident sunlight. ${ }^{[9]}$
Around each photosystem, large amounts of carotenoids and chlorophylls are assembled in antenna complexes that absorb solar light and channel the excitation energy to the reaction centres of the photosystems, greatly increasing the absorption cross section per photosystem and thereby improving their energetic payoff. The energy transfer (ET) mechanism has long been described by semi-classical models that invoke 'hopping' of excited states from molecule to molecule (Fig. 1). ${ }^{[22,23]}$ Recent investigations have found evidence for long-lived (several hundred fs) electronic quantum coherence states in photosynthetic complexes, even at room temperature. These surprisingly long-lived states gave rise to the hypothesis that they play an important role in energy transfer processes or photoprotection. ${ }^{[24-26]}$

The energy finally reaches the special chlorophylls in the reactive centre. These chlorophylls are called P680 (pigment 680) due to their absorption maximum at $680 \mathrm{~nm}$. Excited P680 leads to the initial charge separation into two radicals, a pheophytin anion radical $\left(\mathrm{Pheo}^{-}\right)$and $\mathrm{P}_{680^{+}}$, within a few ps (Scheme 1). In much slower steps, the electron is then transferred to plastoquinone $\left(Q_{B}\right)($ Fig. 1). After a second cycle, the doubly reduced and protonated plastoquinol is released and transports the electrons downstream towards photosystem I.

In turn, the hole of the reaction centre chlorophyll $\mathrm{P}_{680^{+}}$is re-reduced by electrons from the water-oxidizing complex (WOC, also manganese cluster) where water is oxidised to dioxygen and $\mathrm{H}^{+}$in a four-step cycle.[27]

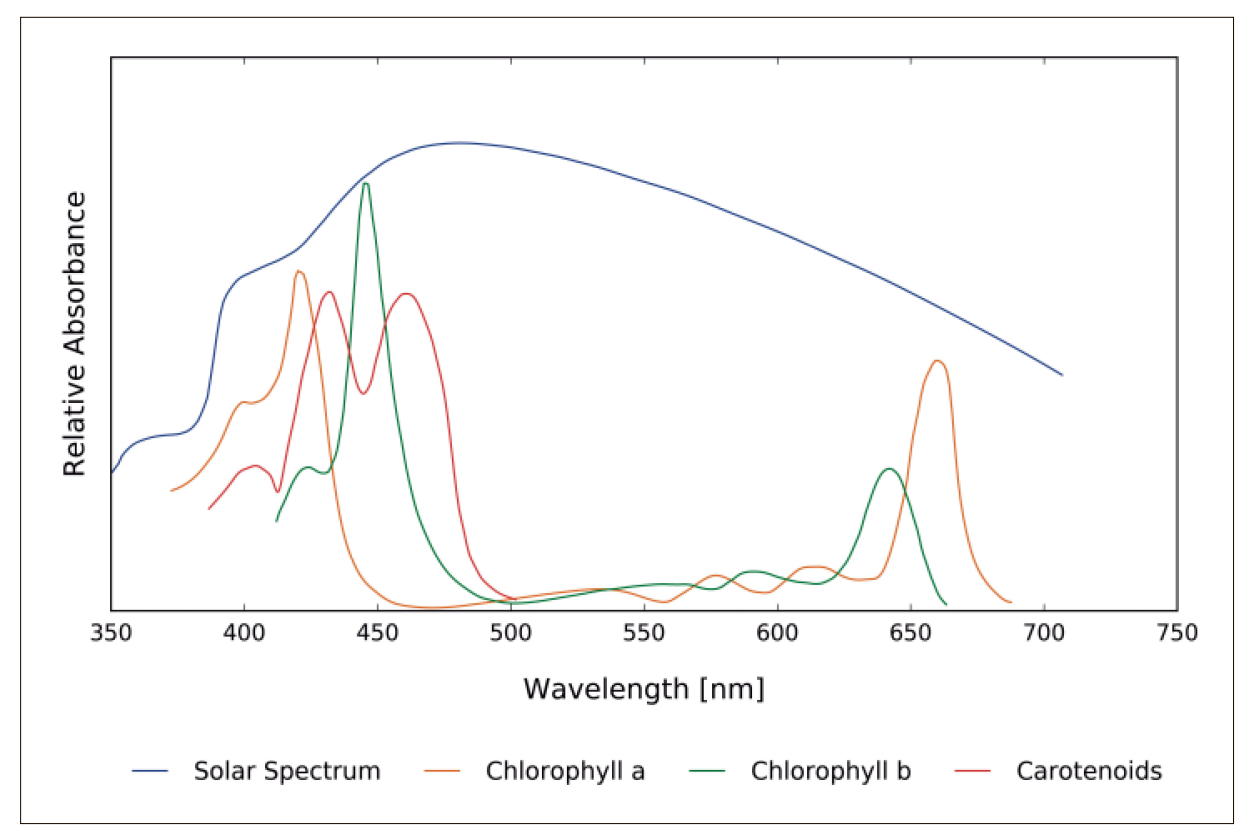

Fig. 2. Intensity of the solar radiation (blue) and absorption by photosynthetic pigments (chlorophyll a: orange, chlorophyll b: green, carotenoids: red) as a function of wavelength. The 'green region' of the spectrum $(480-620 \mathrm{~nm})$ is hardly absorbed. Adapted from ref. [28]. 
The efficient charge separation in the reaction centre is a key factor in this process. This energy-rich state has obviously the distinct tendency to recombine into its ground state, emitting the energy as light (fluorescence) or simply as heat. In the photosystems, charge separation is very fast. ${ }^{[3]}$ The electron and the hole are efficiently separated over a large distance and charge recombination is largely prevented except under conditions of light oversaturation or blocked electron transfer (Fig. 3). Charge separation and its stabilization is also a core step in artificial water splitting. Its longevity and chemical quenching is one of the challenges in the conception of chromophores and coupled reactions.

In short, the conversion efficiency of solar energy to biomass is generally below $1 \%$ and may therefore seem rather low. Nevertheless, photosynthetic sub-processes can be remarkably fast and energy efficient, for instance the transfer of the $a b$ sorbed energy from the antenna complexes to the reactive centres of the photosystems and the subsequent charge separation. Such a remarkable performance is possible because the involved components are arranged in well-defined supramolecular assemblies. In these assemblies, reaction

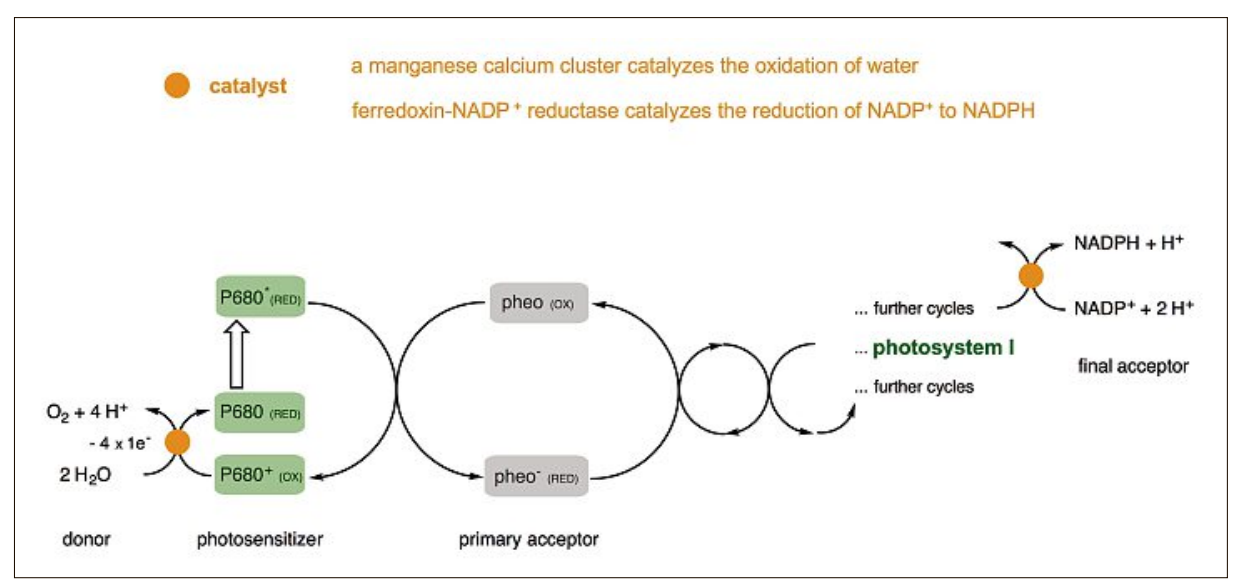

Scheme 1. Overview showing the essential processes in PSII: Absorption and excitation of P680, charge separation in the reaction centre through the formation of the pheophytin radical anion (Pheo-) and $\mathrm{P} 60^{+}$, oxidation of the donor water through the manganese cluster (orange) and subsequent hole filling on P680.

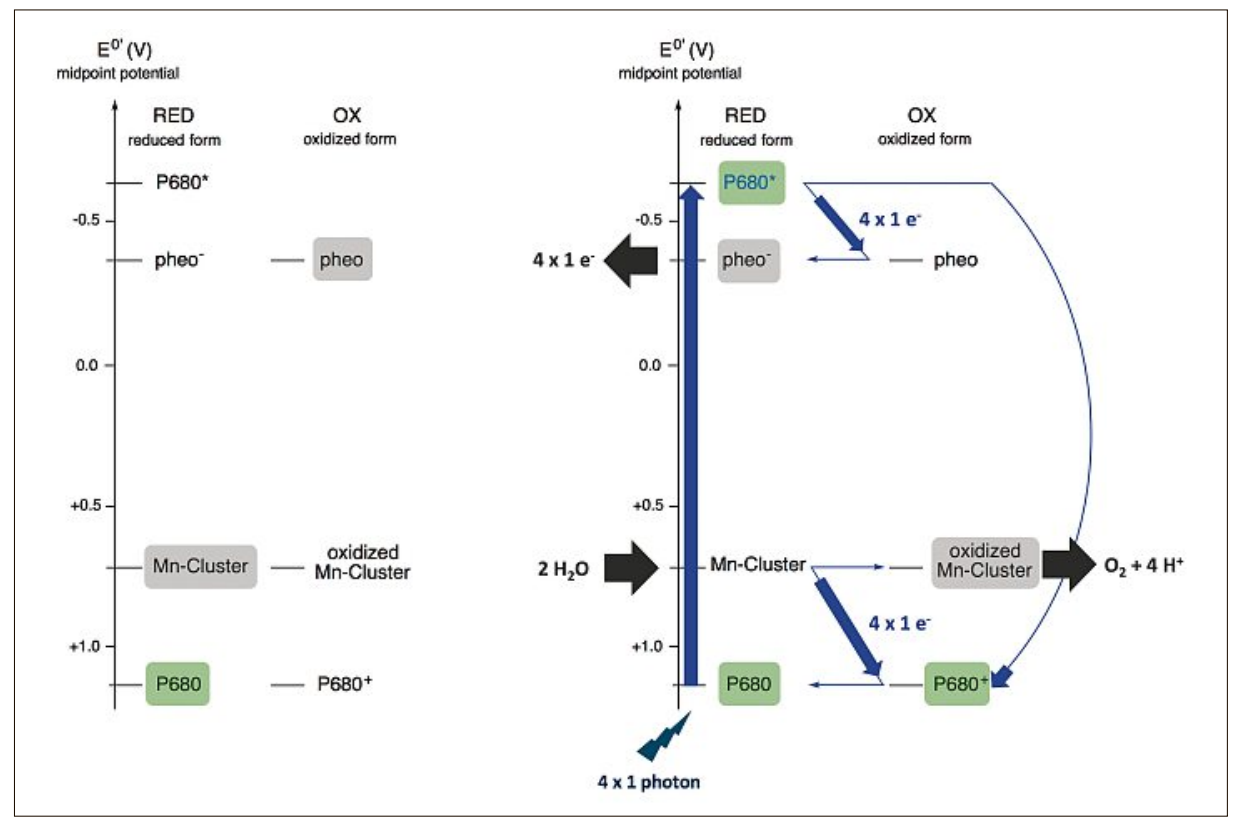

Fig. 3. Redox diagrams of the essential processes in PSII. The left diagram shows the dark adapted photosystem, in which the reaction centre P680 and the manganese cluster prevail in their reduced states, whereas pheophytin is uncharged and oxidised. Right diagram: Upon excitation $\mathrm{P} 680$ becomes a very strong reductant $\left(\mathrm{P} 680^{*}\right)$ that has a midpoint potential low enough to donate an electron to pheophytin, thus giving rise to the initial charge separation. ${ }^{[29]}$ The resulting $\mathrm{P} 680^{+}$is a strong oxidant that is re-reduced to ground state P680 by electrons from the $\mathrm{Mn}$-cluster. In four subsequent steps, the Mn-cluster becomes increasingly oxidised and concomitantly expels $4 \mathrm{H}^{+}$, until it releases one oxygen molecule and binds two molecules of water, whereby the dark adapted state is reconstituted.

partners are in tight contact, whereas the oxidised donors remain carefully separated from the reduced acceptors, thereby preventing inefficient shortcuts. The supramolecular organisation may even facilitate quantum superpositions of excited chlorophylls that substantially enhance the funnelling of excitation energy into the reaction centres.

\section{Aspects of Artificial Photosynthesis}

The reductive equivalents on the plastoquinols are then converted in photosystem I after further excitation processes at $700 \mathrm{~nm}$ to NADPH. PSI is equally welldesigned as PSII and a structural overview is given in Fig. 4. The individual components are explained in the figure caption.

Without going into mechanistic or structural details of PSI, research in artificial photosynthesis, or better in photocatalytic water splitting, aims at mimicking the function of this highly complex protein architecture with small molecules and catalysts. The structural sketch shown in Fig. 4 reveals how challenging such a translation from large to small may be. Still, the artificial systems as outlined below follow conceptually their natural model; light harvesting in dyes is followed by electron/ hole separation and catalytic oxidation/ reduction processes leading to $\mathrm{H}_{2}$ and $\mathrm{O}_{2}$.

In artificial photosynthesis, it is believed that water oxidation is a more complex process as compared to water (proton) reduction since the former is a four- and the latter only a two-electron process. ${ }^{[31]}$ However, differentiation between photoelectrochemical and photocatalytic water splitting has to be made. As mentioned in the introduction, the architectures of feasible, lightdriven water splitting have widely been disputed. Without adding another opinion, two points shall be explicitly stated; i) as in natural photosynthesis, water splitting will not be a fully homogeneous process with all (molecular) components dissolved in water since highly reducing and highly oxidizing species do not coexist. Shortcuts of all kind make such a system unproductive and ii) it is a persistent question if future, non-wired water splitting will be based on materials or molecules. The argument that nature does it with molecules is in favour of the former, the much superior stability of materials for the latter statement. In fact, working systems for both exist, Nocera's artificial leaf, ${ }^{[14]}$ van de Krol's $\mathrm{BiVO} / \mathrm{Si}$ tandem cells or Grätzel's perovskite cell[16] are typical examples for material-based water splitting, wired or non-wired. Sun's ruthenium complexes and others exemplify molecule-based water splitting. ${ }^{[32]}$ With molecule-based catalysts, the two process- 


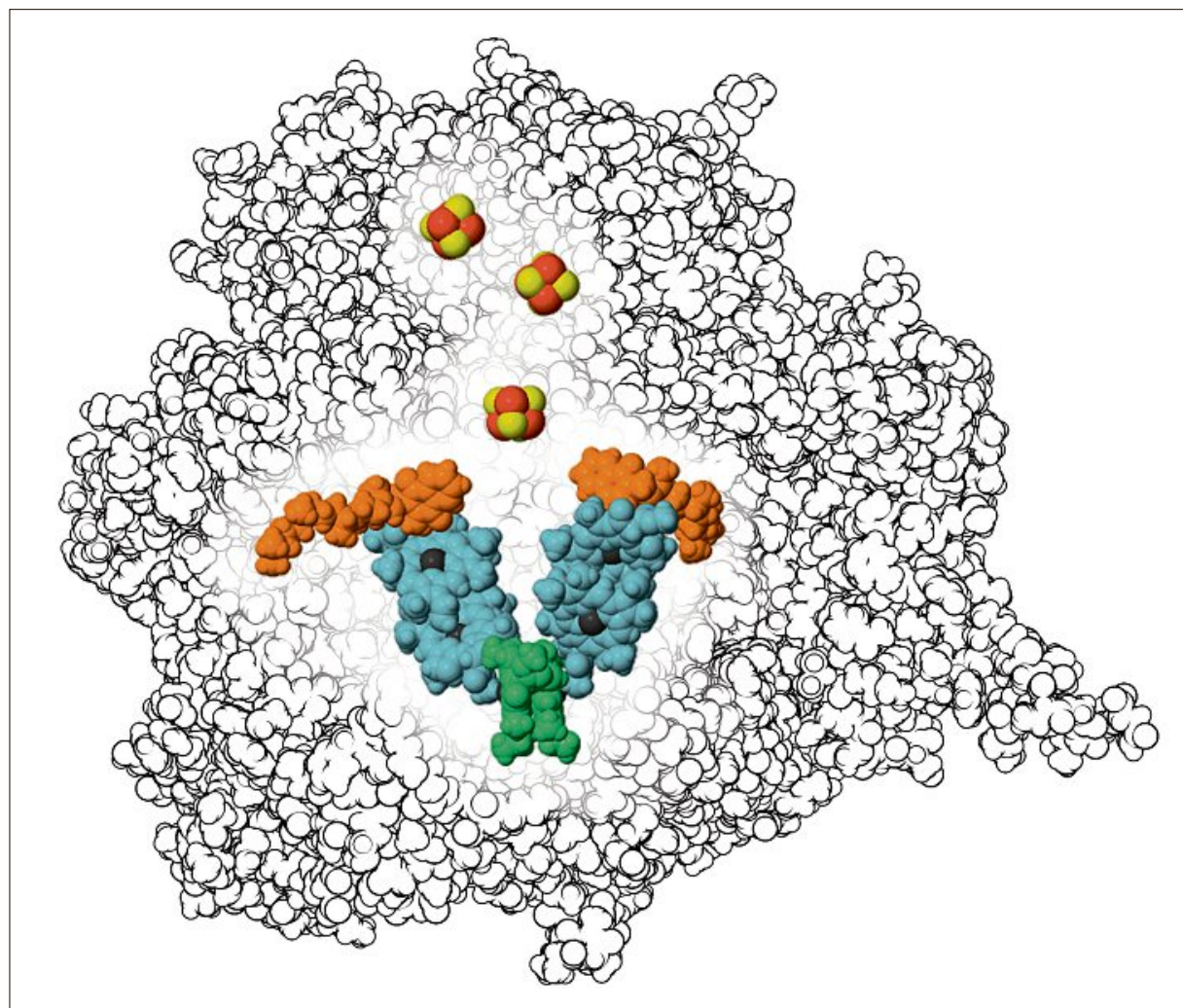

Fig. 4. The reactive centre of photosystem I (PSI) consists of two parallel chlorophyll molecules with slightly different characteristics (light green). Upon excitation, an electron is passed along either of them to pseudosymmetrical chlorophyll branches (light blue) through phylloquinones (orange) and a chain of iron-sulphur clusters (yellow and brown) to water soluble ferredoxin that is located in the fluid inside the inner chloroplast membrane (stroma). The reaction centre of PSI is re-reduced by plastocyanin with electrons from the PSII. Chlorophyll is shown without isoprenoid side chains. The image was created from crystallographic coordinates found in PDB ID: $1 \mathrm{JBO} 0{ }^{[30]}$

es are generally studied separately and in homogeneous solution. The fact that solutions allow detailed kinetic and thermodynamic studies with generally available analytical instruments such as infrared, $\mathrm{UV/vis}$ spectroscopy or electrochemistry is one of the reasons. Obtained data help to understand processes on a molecular level, having relevance to processes on surfaces, where characterization of compounds is much more difficult requiring not easily available instruments.

When separating water splitting in two separate processes, the role of PSII as ultimate electron donor from oxidizing water in proton reduction to $\mathrm{H}_{2} / \mathrm{NADPH}$ is taken over by chemical reductants, so-called sacrificial electron donors (SEDs) since they are used up in photocatalysis thereby limiting the final yields. This is the process we want to have a closer look at in the following section. In Scheme 2, the artificial proton reduction process is compared to (simplified) biological water splitting. In analogy, water oxidation requires a sacrificial electron acceptor, mostly peroxo-disulfate or $\mathrm{Ce}(\mathrm{IV})$ in strongly acidic solution.

A large variety of WRCs have been described in the literature with the majority being based on cobalt, nickel or iron. ${ }^{[33-36]}$ Prominent catalysts are shown in Fig. 5.
Historically, glyoxamato complexes of $\mathrm{Co}^{\mathrm{III}}$ (1) have been studied in detail, although mostly in organic solvents and with low $\mathrm{H}_{2}$ yields. Complex $\mathbf{1}$ is easily prepared with commercially available chemicals. It is a good exercise to identify a few properties that a metal centre in a complex should have to act as a WRC. Assuming that a hydride is the direct precursor to dihydrogen formation, the oxidation state of the metal centre should be compatible with an $\mathrm{M}-\mathrm{H}$ bond. This hydride must not be an acid, i.e. the $\mathrm{p} K$ of the $\mathrm{M}-\mathrm{H}$ entity should be high to make the hydride 'hydridic'. The metal should accommodate at least two electrons in an accessible potential range and electron transfer from a reduced photosensitizer must be fast, e.g. through an outer-sphere process between appropriate orbitals. Following the entatic principle, a regular geometry is probably not ideal since it would demand extensive geometrical (and electronic) rearrangements upon reduction and protonation. Thus, the fastest rates are obtained in a distorted geometry that lies somewhere between the optimal geometries of each oxidation state. Ligands should be redoxinactive since catalysis is thought to take place at the metal rather than on the ligands. According to these arguments, cobalt is a good choice and that's why many studies have been done with it.

For cobalt, we have designed a number of pyridine-based catalysts under these aspects. They are acyclic or cyclic, depending on the weight given to one or the other of the above considerations. Some of the WRCs are shown in Fig. 5.

All these catalysts produce $\mathrm{H}_{2}$ in the presence of a photosensitizer and an SED, typically ascorbic acid or triethanolamine. The elucidation of the mechanism is crucial and basic to improve the catalyst. Taking the simplicity of proton reduction into account, $2 \mathrm{H}^{+}+2 \mathrm{e}^{-} \rightarrow \mathrm{H}_{2}$ (the 'easiest' reaction one can imagine), one or more 'reasonable' schemes, composed of sev-

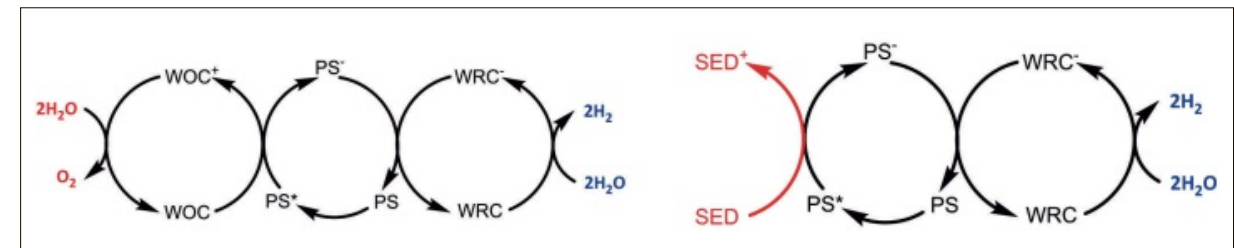

Scheme 2. Separation of a full water splitting cycle (left) into the reductive process only (right). Water-oxidizing catalyst (WOC), water-reduction catalyst (WRC), sacrificial electron donor (SED), photosensitizer (PS). Water oxidation as the electron donating process is replaced by an SED.

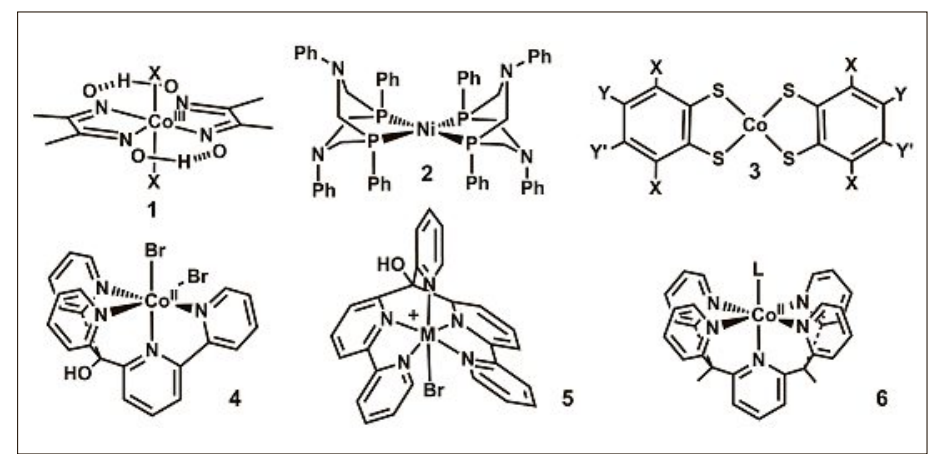

Fig. 5. Typical water reduction catalysts: upper row, the dimethyl-glyoxamatoCo'll complex 1, the Dubois catalyst $2^{[37]}$ and a dithiolene system from Eisenberg et al. ${ }^{[38] ;}$ lower row, tetraand penta-pyridyl catalysts developed in our lab and by Chang et al. ${ }^{[39-41]}$ 
Scheme 3. One or two or three or even more reasonable mechanisms for light-driven, catalytic proton reduction. Which is the correct one? Electrons in the scheme are provided by the excited PS after its reductive quenching with an SED.

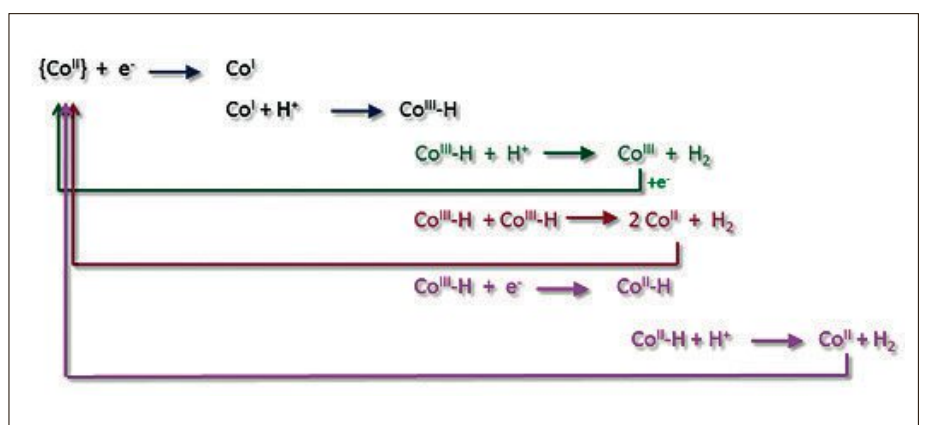

eral elementary steps, can immediately be drawn (Scheme 3).

Which of the reaction schemes is now the most likely one, or are there equally reasonable, further alternatives? Think about it! After excitation of the photosensitizer, we assume a so-called reductive quenching of its excited state by electrons from the SED (or from the WOC in a complete system), followed by electron transfer to the $\mathrm{Co}^{\mathrm{II}}$ of the WRC. In principle, the reverse sequence, oxidative quenching of the excited state (electron transfer to the WRC) and subsequent reduction of the oxidized PS is reasonable as well; in fact, in biological photosynthesis, the excited special pair P680* in PSII is oxidatively quenched within about $1 \mathrm{ps}$, whereas its re-reduction takes much longer, lasting as long as 200 ns. ${ }^{[3]}$ Such enormous rates are possible since all components are fixed in close proximity and diffusion does not limit the rate. This is different in homogeneous catalysis in which the maximal achievable rate constants are diffusion limited and in the range of $5 \cdot 10^{9} \mathrm{M}^{-1} \mathrm{~s}^{-1}$.

Does the difference matter at all? Yes, it does, since each step is coupled to kinetic and thermodynamic parameters. To optimize a system, the respective constants and the underlying mechanisms have to be known. We found with ultra-fast IR spectroscopy that reductive quenching takes place indeed. We determined the corresponding rate constants. They are in general $>10^{8} \mathrm{M}^{-1} \mathrm{~s}^{-1}$, thus, very fast. However, the highly dilute solutions make bimolecular reactions slow. We have elucidated full mechanistic details over the years and reported about the kinetic and thermodynamic numbers. ${ }^{[42,43]}$

Then, in a second step, the electron is transferred to the $\mathrm{WRC}$, i.e. $\mathrm{Co}^{\mathrm{II}}$ is reduced to $\mathrm{Co}^{\mathrm{I}}$. Is this a rate-limiting step or is it fast in comparison to the other steps? It is very fast, and almost diffusion controlled. Still, one has to consider that this is a $2^{\text {nd }}$ order reaction. Despite high rates, the low WRC concentration will result in a slow electron transfer step, leading potentially to decomposition of the once reduced photosensitizer. We have now a $\mathrm{Co}^{\mathrm{I}}$ and can assume its protonation to $\mathrm{Co}^{\text {III-H}}-\mathrm{H}$. This hydride might be the direct precursor to
$\mathrm{H}_{2}$ formation, depending on its nature: does it exhibit a high hydricity, or does it rather react as an acid $\left(\mathrm{Co}^{\mathrm{III}}-\mathrm{H} \leftrightarrow \mathrm{Co}^{\mathrm{I}}\right.$ $+\mathrm{H}^{+}$or $\mathrm{Co}^{\mathrm{III}}-\mathrm{H} \leftrightarrow \mathrm{Co}^{\mathrm{III}}+{ }^{\prime} \mathrm{H}^{-}$), what is its $\mathrm{p} K$ value? ${ }^{[44]}$ In a third step, we now might assume protonation of the hydride to yield $\mathrm{H}_{2}$ and a $\mathrm{Co}^{\mathrm{III}}$ complex (green track in Scheme 3). However, detailed kinetic studies showed that this is not necessarily the case. Instead, the $\mathrm{Co}^{\mathrm{III}}-\mathrm{H}$ is again reduced to $\mathrm{Co}^{\mathrm{II}}-\mathrm{H}$ (pink track) which yields a 'real' $\mathrm{Co}^{\mathrm{II}}$ hydride which is only then protonated to $\mathrm{Co}^{\mathrm{II}}$ and $\mathrm{H}_{2}{ }^{[42]}$ There are further 'reasonable' routes (red track). The knowledge of the prevailing reaction sequence (there may be competing ones) is decisive for improving/developing efficient catalysts. It took us years to deduce precise mechanisms for water reduction with one system. The mechanism of a different catalyst may be different again. Finally, how stable are the participating complexes and molecules under photochemical conditions? They are generally in energetically high-lying states and therefore prone to decomposition. Based on mechanistic knowledge, it is possible to improve catalysts. We proceeded from acyclic poly-pyridyl systems to macrocyclic ones, resembling porphyrins but being assembled from pyridines. A selection of these catalysts and the structures of their complexes are shown in Scheme 4.
All proton-reduction catalysts together with $\left[\mathrm{Ru}(\text { bpy })_{3}\right]^{2+}$ or $\left[\operatorname{Re}\left(\text { py) }(\text { bpy })(\mathrm{CO})_{3}\right]^{+}\right.$ as photosensitizers and ascorbic acid as SED reach substantial turnover numbers as high as $80 ' 000 \mathrm{H}_{2} / \mathrm{Co}$ until $\mathrm{H}_{2}$ evolution ceases. Even this performance is still far from what is requested for an applicable system. In this brief section, only mechanistic/kinetic factors are touched. Thermodynamic factors such as overpotentials and driving forces complicate the system further but are not considered in here. Their implementation in an ultimate design towards a long-term working system is still essential. Finally, one should not forget that a system with an SED is completely academic and water reduction must be chemically or physically brought down to a level where it can be combined with water oxidation, along whatever architecture.

In essence, even the simplest reaction $2 \mathrm{H}^{+}+2 \mathrm{e}^{-} \rightarrow \mathrm{H}_{2}$ is in fact a highly complex system, especially when driven by light. Artificial photocatalytic systems involve interesting and beautiful molecules and require a fascinating multitude of aspects to be fully understood for finding a final solution. Going back to the beginning of this article, our studies of artificial photosynthetic systems have led us to appreciate the natural system, which is far more sophisticated but functions in a perfect, situationadapted way. It has efficiently powered life on our planet for billions of years.

\section{Acknowledgments}

Financial support from the Swiss National Science Foundation (Sinergia project CRSII2_160801/1), the University of Zürich and the 'Light to Chemical Energy Conversion' project is gratefully acknowledged.

Received: October 2, 2017

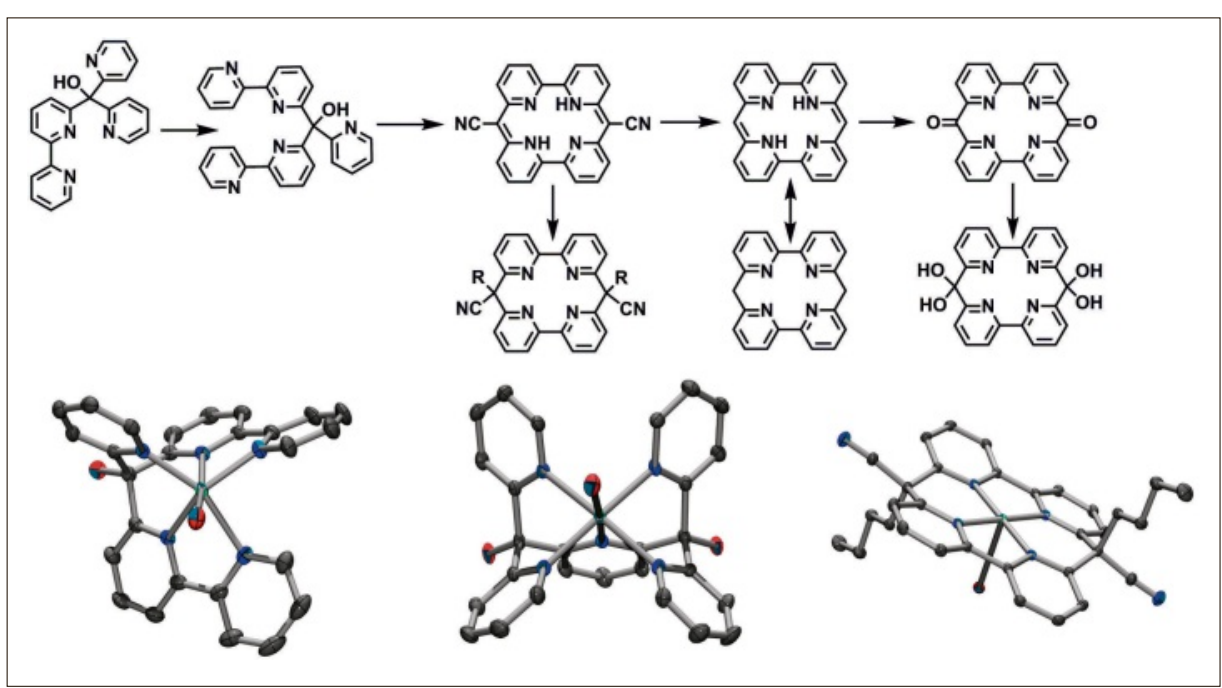

Scheme 4. Poly-pyridyl ligand development from acyclic to cyclic systems (top row); a number of $\mathrm{X}$-ray structures of Co"l-complexes with acyclic and cyclic poly-pyridyl ligands. 
[1] B. B. Buchanan, W. Gruissem, R. L. Jones, 'Biochemistry and Molecular Biology of Plants', 2nd ed., John Wiley \& Sons, 2015.

[2] R. E. Blankenship, 'Molecular Mechanisms of Photosynthesis', 2nd ed., John Wiley \& Sons, Chichester, 2014

[3] B. A. Diner, F. Rappaport, Annu. Rev. Plant. Biol. 2002, 53, 551.

[4] J. Barber, Phil. Trans. Royal Soc. A 2007, 365, 1007.

[5] H. Michel, Angew. Chem. Int. Ed. 2012, 51, 2516.

[6] J. W. Ponton, J. Clean. Prod. 2009, 17, 896.

[7] A. Ruban, 'The Photosynthetic Membrane: Molecular Mechanisms and Biophysics of Light Harvesting', John Wiley \& Sons, Ltd, Chichester, UK, 2012.

[8] R. S. Knox, W. W. Parson, Bba-Bioenergetics 2007, 1767, 1189.

[9] X.-G. Zhu, S. P. Long, D. R. Ort, Curr. Opin. Biotechnol. 2008, 19, 153.

[10] M. T. F. Piedade, W. J. Junk, S. P. Long, Ecology 1991, 72, 1456.

[11] H. Lambers, F. S. Chapin, T. L. Pons, 'Plant Physiological Ecology', 2nd ed., Springer New York, New York, 2008.

[12] R. E. Blankenship, D. M. Tiede, J. Barber, G. W. Brudvig, G. Fleming, M. Ghirardi, M. R. Gunner, W. Junge, D. M. Kramer, A. Melis, T. A. Moore, C. C. Moser, D. G. Nocera, A. J. Nozik, D. R. Ort, W. W. Parson, R. C. Prince, R. T. Sayre, Science 2011, 332, 805.

[13] N. Armaroli, V. Balzani, Angew. Chem. Int. Ed. 2007, 46, 52.

[14] D. G. Nocera, Acc. Chem. Res. 2012, 45, 767.

[15] M. Grätzel, Nature 2001, 414, 338.

[16] J. S. Luo, J. H. Im, M. T. Mayer, M. Schreier, M. K. Nazeeruddin, N. G. Park, S. D. Tilley, H. J. Fan, M. Grätzel, Science 2014, 345, 1593.
[17] B. A. Pinaud, J. D. Benck, L. C. Seitz, A. J. Forman, Z. B. Chen, T. G. Deutsch, B. D. James, K. N. Baum, G. N. Baum, S. Ardo, H. L. Wang, E. Miller, T. F. Jaramillo, Energy Environ. Sci. 2013, 6, 1983.

[18] K. N. Ferreira, T. M. Iverson, K. Maghlaoui, J. Barber, S. Iwata, Science 2004, 303, 1831.

[19] M. O. Senge, A. A. Ryan, K. A. Letchford, S. A. MacGowan, T. Mielke, Symmetry 2014, 6, 781 .

[20] W. P. Bricker, P. M. Shenai, A. Ghosh, Z. T. Liu, M. G. M. Enriquez, P. H. Lambrev, H. S. Tan, C. S. Lo, S. Tretiak, S. Fernandez-Alberti, Y. Zhao, Sci. Rep. UK 2015, 5, xxx.

[21] G. D. Scholes, T. Mirkovic, D. B. Turner, F Fassioli, A. Buchleitner, Energy Environ. Sci. 2012, 5, 9374

[22] Z. Gokhale, R. T. Sayre, in 'The Chlamydomonas Sourcebook', Vol. 2, 2nd ed., Eds. D. Stern, E. H. Harris, G. Witman, Academic, London, 2009, p. 573.

[23] G. S. Engel, T. R. Calhoun, E. L. Read, T. K. Ahn, T. Mancal, Y. C. Cheng, R. E. Blankenship, G. R. Fleming, Nature 2007, 446, 782.

[24] M. O. Scully, K. R. Chapin, K. E. Dorfman, M. B. Kim, A. Svidzinsky, Proc. Natl. Acad. Sci. USA 2011, 108, 15097.

[25] K. E. Dorfman, D. V. Voronine, S. Mukamel, M. O. Scully, Proc. Natl. Acad. Sci. USA 2013, 110, 2746.

[26] F. Fassioli, R. Dinshaw, P. C. Arpin, G. D. Scholes, J. R. Soc. Interface 2014, 11, xxx.

[27] W. Hillier, J. Messinger, in 'Photosystem II', Vol. 22, Eds. T. J. Wydrzynski, J. A. Freeman, K. Satoh, Springer, Dordrecht, 2005, p. 567, DOI: 10.1007/1-4020-4254-X_26.

[28] D. Voet, J. G. Voet, 'Biochemistry', 3rd ed., Wiley, Hoboken NJ, 2004

[29] V. V. Klimov, A. V. Klevanik, V. A. Shuvalov, A. A. Kransnovsky, FEBS Lett. 1977, 183.
[30] P. Jordan, P. Fromme, H. T. Witt, O. Klukas, W. Saenger, N. Krauss, Nature 2001, 411, 909.

[31] L. Hammarstrom, Acc. Chem. Res. 2015, 48, 840.

[32] L. Wang, K. Fan, H. Chen, Q. Daniel, B. Philippe, H. Rensmo, L. C. Sun, Catal. Today 2017, 290, 73.

[33] Z. J. Han, R. Eisenberg, Acc. Chem. Res. 2014, 47, 2537.

[34] K. S. Joya, Y. F. Joya, K. Ocakoglu, R. van de Krol, Angew. Chem. Int. Ed. 2013, 52, 10426.

[35] V. Artero, M. Chavarot-Kerlidou, M. Fontecave, Angew. Chem. Int. Ed. 2011, 50, 7238.

[36] F. Gloaguen, T. B. Rauchfuss, Chem. Soc. Rev. 2009, 38, 100 .

[37] M. L. Helm, M. P. Stewart, R. M. Bullock, M. R. DuBois, D. L. DuBois, Science 2011, 333, 863.

[38] A. Das, Z. J. Han, M. G. Haghighi, R. Eisenberg, Proc. Natl. Acad. Sci. USA 2013, 110, 16716.

[39] Y. J. Sun, J. P. Bigi, N. A. Piro, M. L. Tang, J. R. Long, C. J. Chang, J. Am. Chem. Soc. 2011, 133, 9212.

[40] B. Probst, A. Rodenberg, M. Guttentag, P. Hamm, R. Alberto, Inorg. Chem. 2011, 50, 3404.

[41] E. Joliat, S. Schnidrig, B. Probst, C. Bachmann, B. Spingler, K. K. Baldridge, F. von Rohr, A. Schilling, R. Alberto, Dalton Trans. 2016, 45, 1737

[42] A. Rodenberg, M. Orazietti, B. Probst, C. Bachmann, R. Alberto, K. K. Baldridge, P. Hamm, Inorg. Chem. 2015, 54, 646.

[43] C. Bachmann, B. Probst, M. Guttentag, R Alberto, Chem. Commun. 2014, 50, 6737.

[44] E. S. Wiedner, M. B. Chambers, C. L. Pitman, R. M. Bullock, A. J. M. Miller, A. M. Appel, Chem. Rev. 2016, 116, 8655. 